\title{
Usefulness of Radiofrequency Ablation with the PVT-350BTP Probe for the treatment of Hepatocellular Carcinoma
}

\author{
Naoki Hotta*, Kazuo Masuko \\ Department of Internal Medicine, Division of Hepatology, Masuko Memorial Hospital, Nagoya, Japan \\ E-mail:"naokiki@aol.com.
}

Received September 5, 2011; revised September 13, 2011; accepted September 14, 2011

\begin{abstract}
Radiofrequency ablation (RFA) has been clinically employed in Japan, and its usefulness in the treatment of hepatocellular carcinoma (HCC) has been reported by many researchers. In the present study, the convex puncture probe PVT-350BTP, rather than a conventional convex probe, was used for RFA for the treatment of HCC and its usefulness was assessed. At the same time, evaluation of the therapeutic effect using ultrasound contrast agent was investigated. The subjects were 20 patients (16 men and 4 women) with 20 HCC nodules as confirmed by contrast CT or abdominal angiography. No nodules were $\leq 1.0 \mathrm{~cm}$ in diameter, 12 were $1.1 \mathrm{~cm}-2.0 \mathrm{~cm}$, and 8 were $2.1 \mathrm{~cm}-3.0 \mathrm{~cm}$. An Aplio diagnostic ultrasound system (Toshiba Medical Systems, Tokyo, Japan) and the PVT-350BTP probe (Toshiba) were employed for RFA. The PVT-350BTP supports puncture angles of 55, 70, 85, and 100 degrees. By mounting various types of puncture adapters on the probe, $13 \mathrm{G}$ to $22 \mathrm{G}$ puncture needles can be used. The needle can easily be attached and detached by operating a lever. Contrast echo studies with Levovist were performed before and after RFA, and the results were evaluated using the Advanced Dynamic Flow (ADF) imaging technique. Puncture was performed successfully using this probe for 20 cases with 20 nodules. It was confirmed that RFA with the PVT-350BTP permits percutaneous treatment to be performed more reliably than with a conventional probe.
\end{abstract}

Keywords: Ultrasound, RFA, HCC , PVT-350BTP

\section{Introduction}

Radiofrequency ablation (RFA)[1-3] has been clinically employed in Japan, and its usefulness in the treatment of hepatocellular carcinoma (HCC) has been reported by many researchers. RFA can be performed in various ways: percutaneously, laparoscopically, via a small incision, or intraoperatively. In our department, we generally perform echo-guided percutaneous RFA, and we are also attempting RFA with 3D echo imaging.[4] In the present study, we employed a convex puncture probe, the PVT350BTP, rather than a conventional convex probe, for RFA for the treatment of HCC and assessed its usefulness. At the same time, evaluation of the therapeutic effect using ultrasound contrast agent was investigated.

\section{Subjects and Methods}

The subjects were 20 patients ( 16 men and 4 women) with 20 HCC nodules as confirmed by contrast CT or abdominal angiography. Their age range was 37 years to 83 years, with an average age of 65.9 years. All patients were positive for $\mathrm{HCV}$ antibody. sixteen patients were Child-Pugh class A and 4 were class B. No nodules were $\leq 1.0 \mathrm{~cm}$ in diameter, 12 were $1.1 \mathrm{~cm}-2.0 \mathrm{~cm}$, and 8 were $2.1 \mathrm{~cm}-3.0 \mathrm{~cm}$. Prior to the study, informed consent to participate in the study was obtained from each patient.

\subsection{System and Probe Used}

An Aplio diagnostic ultrasound system (Toshiba Medical Systems, Tokyo, Japan) and the PVT-350BTP probe (Toshiba) were employed. This probe supports four puncture angles: 55 degrees, 70 degrees, 85 degrees, and 100 degrees. By mounting various types of puncture adapters on the probe, $13 \mathrm{G}$ to $22 \mathrm{G}$ puncture needles can be used. The needle can easily be attached and detached 
by operating a lever. With this probe, the tip of the needle or electrode is always located on the central axis of the probe, and the needle or electrode can therefore be observed even after it has been inserted into the patient's body (Figure 1).

\subsection{Ultrasound Contrast Agent and Imaging Technique}

Levovist $^{\mathrm{TM}}$ (Nihon Schering Company, Osaka, Japan) was used, with the bolus injection of $7 \mathrm{~mL}$ of Levovist at a concentration of $300 \mathrm{mg} / \mathrm{mL}$ via a peripheral vein at a rate of $1 \mathrm{~mL} / \mathrm{s}$. Advanced Dynamic Flow (ADF) was employed as the contrast mode [5]. The injection of contrast agent was followed by a $10-\mathrm{mL}$ physiological saline flush. The imaging phases were specified as follows: the early arterial phase (10 to 40 seconds after the injection of contrast agent), the late vascular phase (41 seconds to 90 seconds after injection), and the post-vascular phase (5 minutes after injection). Observation with continuous transmission was performed up to 40 seconds after injection, followed by intermittent transmission (with an imaging interval of 1.0 second) for the next 50 seconds. In the post-vascular phase, observation was performed using intermittent transmission (with an imaging interval of 1.5 seconds). The focus was set at the inferior margin of the tumor.

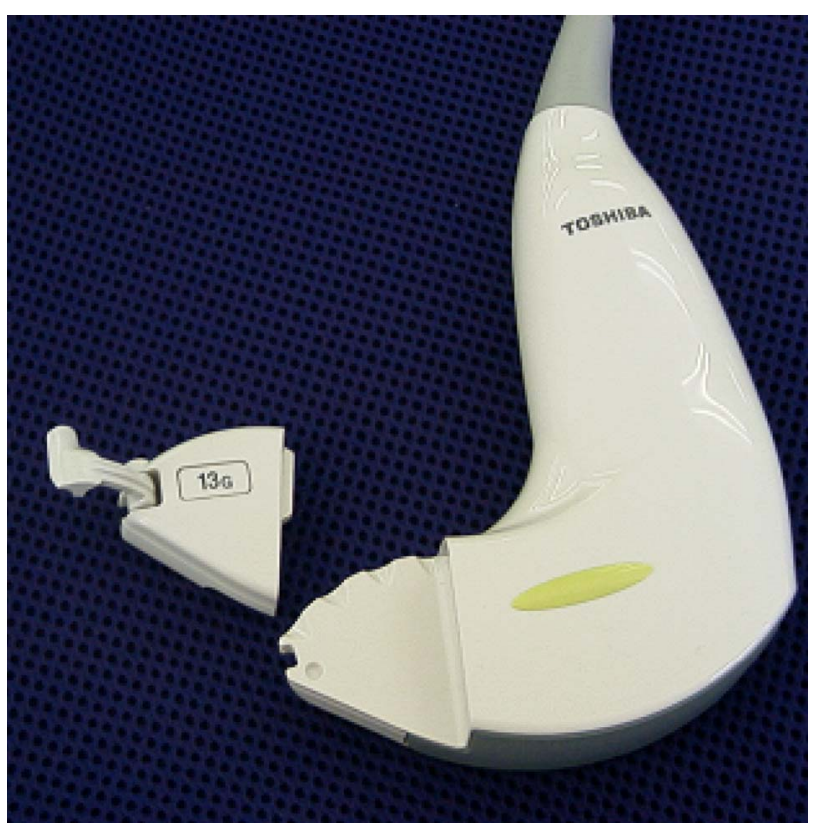

Figure 1. The PVT-350BTP supports four puncture angles: 55 degrees, 70 degrees, 85 degrees, and 100 degrees. It has only one needle slot, but $13 \mathrm{G}$ to $22 \mathrm{G}$ needles can be used by adjusting the screw.

\subsection{Evaluation of Therapeutic Effect}

The therapeutic effect was evaluated using an Aquilion helical CT scanner (Toshiba) with the slice thickness set to $7 \mathrm{~mm}$. The contrast agent Iopamiron (Nihon Schering) was injected at a concentration of $70 \mathrm{mg} / \mathrm{mL}$. The injection volume was $100 \mathrm{~mL}$.

\subsection{RFA Device}

The Cool-tip RF system (Radionics, Burlington, MA) and a single-type needle electrode $(17 \mathrm{G})$ were used for treatment. The electrode was inserted into the tumor and ablation was performed while maintaining the impedance at $90 \mathrm{~W}$ or less. The duration of a single ablation was 12 minutes. To evaluate the therapeutic effect, contrast echo and dynamic CT studies were performed 3 days or 4 days after treatment.

Two representative cases are presented below.

Case 1: A 67-year-old man with chronic hepatitis $\mathrm{C}$ who had been receiving treatment at a local hospital was referred to our department in June 2006 for evaluation due to an increase in the AFP value. Plain US (B-mode) imaging showed a hypoechoic area measuring $1.5 \mathrm{~cm}$ in diameter (Figure 2(a)). In contrast echo imaging, the corresponding area was enhanced in the early arterial phase in ADF mode (Figure 2(b)) and was less strongly enhanced than the hepatic parenchyma in the post-vascular phase (Figure 2(c)). RFA was performed using a Radionics Cool-tip needle electrode at a puncture angle of 100 degrees (Figure 2(d)). The duration of ablation was 12 minutes. The procedure was performed successfully, with no side effects. Four days after treatment, the presence of an adequate safety margin was confirmed by $\mathrm{CT}$ and contrast echo examinations. It was judged that no further RFA procedures were required, and the patient was discharged from the hospital.

Case 2: A 78-year-old woman was referred to our department for treatment of a hepatic tumor. She had been receiving treatment for hepatitis $\mathrm{C}$ at a local hospital. Her family history and previous medical history were otherwise unremarkable. In October 2006, an increased tumor marker value was found in routine blood testing and a hepatic tumor was detected by CT scanning, and the patient was referred to our department. In contrast CT studies, a low-density area was observed in S3 in the pre-contrast phase. The corresponding area was enhanced in the early arterial phase and appeared as a low-density area in the late vascular phase. The patient refused surgery or angiography and expressed a strong preference for RFA. Informed consent was obtained from the patient and her family, and RFA was performed. Contrast echo was used to confirm the exact distance 


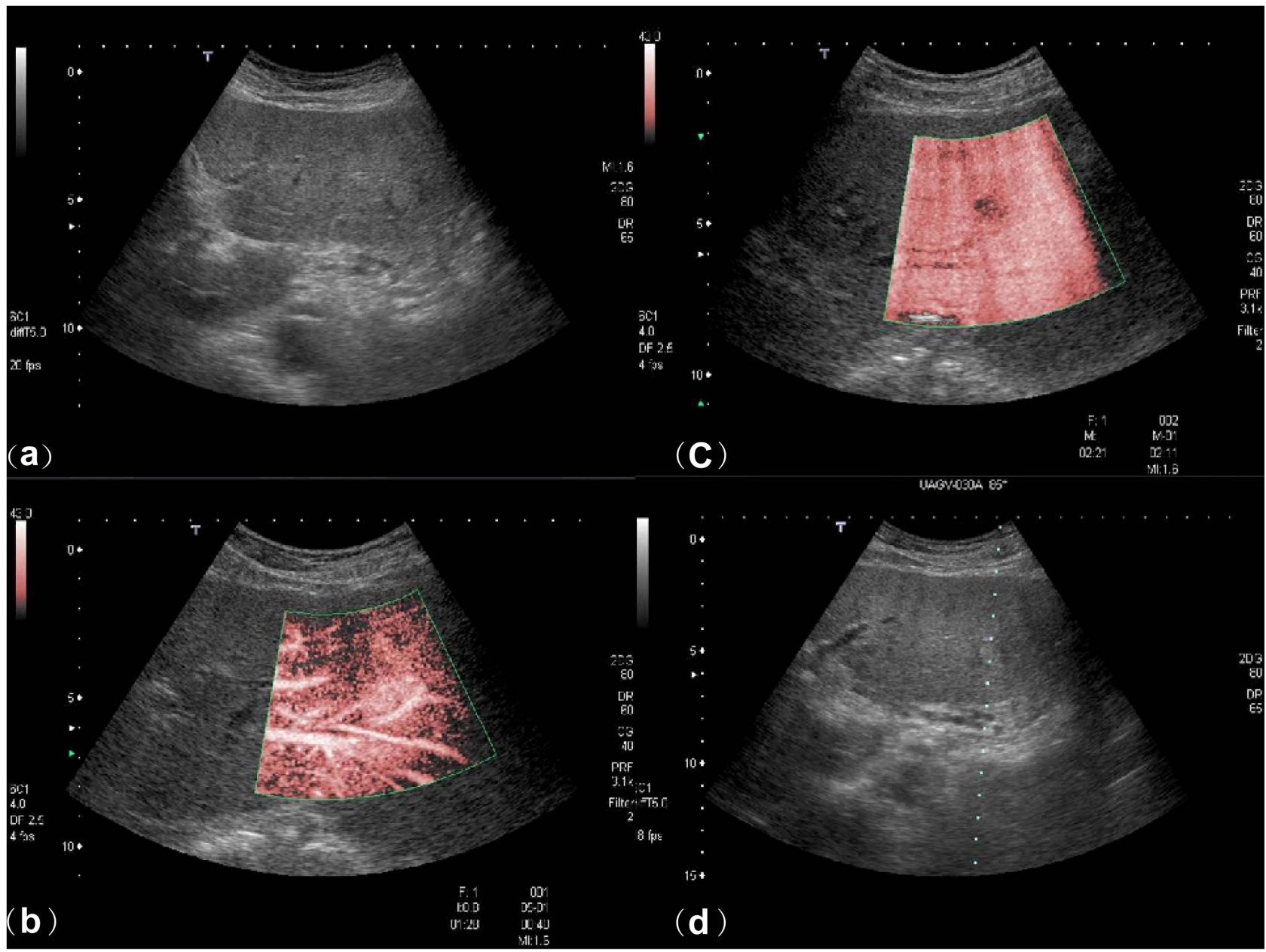

Figure 2. 67-year-old man. A hypoechoic area measuring $1.5 \mathrm{~cm}$ in diameter is seen in a plain US study (B-mode image) (a). The corresponding area is contrast enhanced in the early arterial phase in ADF mode (b), and is less strongly enhanced than the hepatic parenchyma in the post-vascular phase (c). RFA was performed without side effects by using a puncture angle of 100 degrees (d).
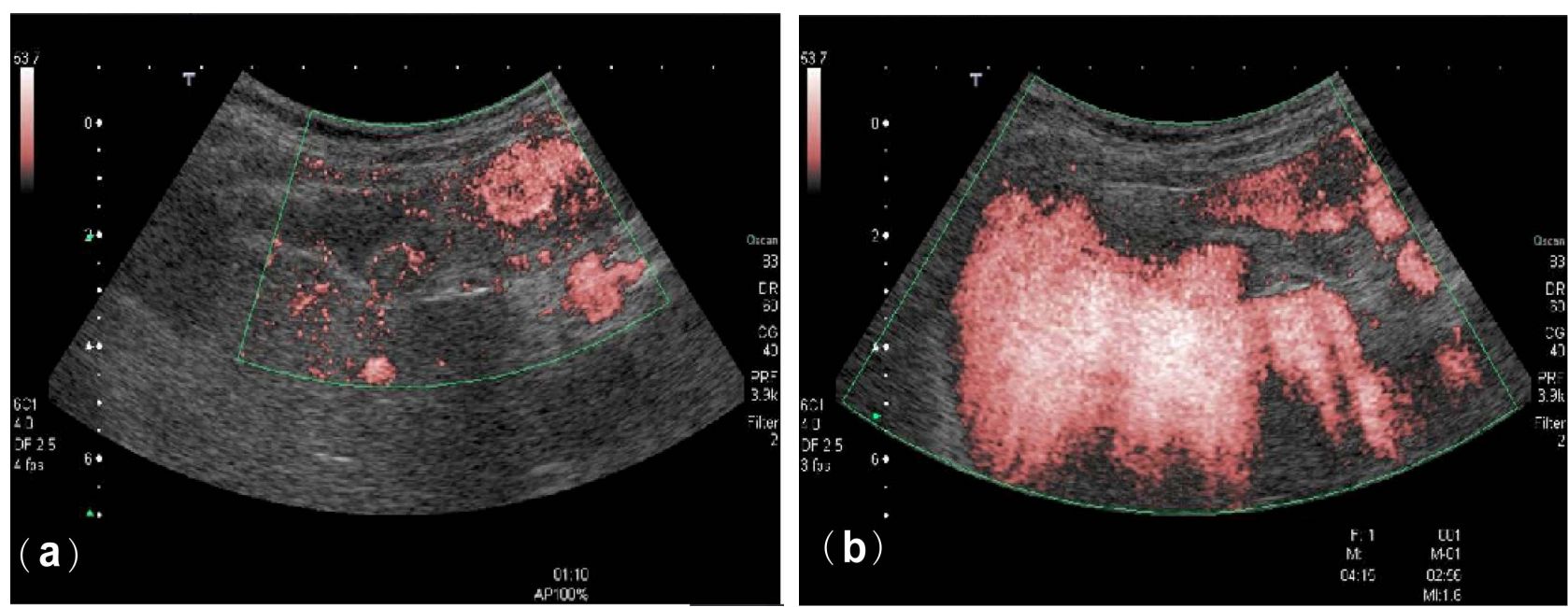

Figure 3. 78-year-old woman. The tumor is enhanced in the early arterial phase in ADF mode (a). In the post-vascular phase, blood flow in the tumor is less than that in the hepatic parenchyma (b). 

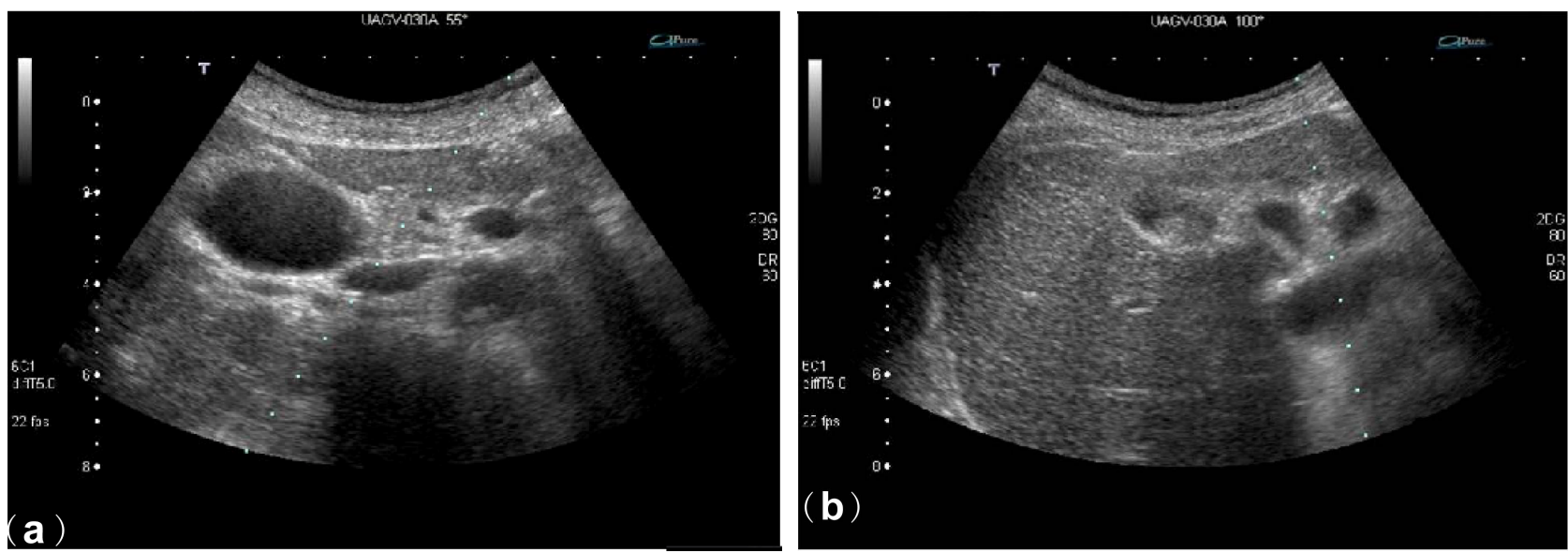

Figure 4. There was a risk of injuring the gallbladder in RFA at a puncture angle of 55 degrees (a). By using a puncture angle of 100 degrees, RFA was performed without side effects (b).

between the tumor and the gallbladder. The tumor was enhanced in the early arterial phase in ADF imaging (Figure 3(a)). In the post-vascular phase, blood flow in the tumor was less than in the hepatic parenchyma (Figure 3(b)). The distance from the gallbladder was judged to be sufficient. At a puncture angle of 55 degrees (Figure 4(a)), however, there was a risk of injuring the gallbladder. A puncture angle of 100 degrees was therefore selected, and the RFA procedure was performed successfully without side effects (Figure 4(b)) [6]. In contrast echo and dynamic CT examinations performed after RFA, no residual blood flow was observed within the lesion and the presence of an adequate safety margin was confirmed. It was judged that no further RFA procedures were required.

\section{Results}

Puncture for RFA was successfully performed for 20 cases with 20 nodules using the PVT-350BTP. In 1 case with a nodule in S8, a micro-convex probe was used instead of the PVT-350BTP. The PVT-350BTP could have been used in this case if artificial pleural effusion had been employed [7], but we demonstrated in a previous study that the micro-convex probe permits RFA to be performed successfully without artificial pleural effusion for the treatment of HCC nodules in S8, and we therefore decided to use the micro-convex probe in this case.[8] In the contrast echo examinations performed before the RFA procedure in order to evaluate the therapeutic effect, blood flowing into the tumor or contrast enhancement of the tumor was observed in the early arterial phase in every case. After the RFA procedure, no blood flowing into the tumor was observed in the early arterial phase in any of the cases. In the post-vascular phase, no supplying vessels were observed and the lesion was seen as a per- fusion defect. In dynamic CT as well, no residual blood flow was observed in the early phase and the presence of an adequate safety margin was confirmed in the late phase in all cases. The evaluation results obtained by contrast echo agreed with those obtained by dynamic CT, although the number of cases was small.

\section{Discussion}

Remarkable advances have been made in diagnostic ultrasound systems in recent years, and echo-guided RFA is employed at many institutions. The ultrasound probe PVT-350BTP can provide images with the same scanning angle as the standard convex probe supplied with the Aplio system. As a result, scanning for treatment planning and scanning for the actual puncture procedure can be performed using the same probe. Although we reported in a previous study that the micro-convex probe is useful for the treatment of HCCs located immediately below the diaphragm, treatment using the PVT- 350BTP was judged to be difficult in 1 case in the present study. For an HCC in the left lobe of the liver, however, RFA could be performed safely with this probe by using a puncture angle of 100 degrees. In addition, RFA could also be performed safely for a tumor located near the gallbladder by using a puncture angle of 100 degrees. This puncture angle also permits RFA to be performed safely for tumors adjacent to the intestines, for which RFA with artificial ascites or laparoscopic RFA are conventionally employed. The PVT-350BTP supports contrast mode, and we therefore employed contrast echo for vascular studies. Since the approval of the ultrasound contrast agent Levovist for clinical use at the end of September 1999, contrast echo with Levovist has been replacing echo-guided intra-arterial $\mathrm{CO}_{2}$ injection following intra-arterial catheterization.[9] There have been 
numerous reports on the usefulness of percutaneous contrast echo with Levovist in the detection and differential diagnosis of hepatic tumors and in the evaluation of therapeutic effects.[10-12] This is a noninvasive method and can be applied to patients with impaired renal function or those who are allergic to the contrast agents that are used in dynamic CT, X-ray angiography, or MRI. One disadvantage is that this method cannot visualize tumors located $10 \mathrm{~cm}$ or more from the liver surface because the acoustic pressure does not increase. In the present study, none of the target tumors were located $10 \mathrm{~cm}$ or more from the liver surface, and evaluation of the therapeutic effect was successfully performed in all cases. In evaluating the therapeutic effect of RFA, determining the presence or absence of residual tumor is the main objective. If an area showing blood flow in the pretreatment contrast echo study appears as an avascular area in the post-treatment contrast echo study, the tumor is judged to be necrotic. This method is also able to detect residual small blood flow that may not be detectable by CT. Even for the nodule that was located near the gallbladder and for which the distance between the nodule and the gallbladder could not be measured accurately by $\mathrm{CT}$ or plain US, the use of ultrasound contrast agent permitted the distance to be measured more accurately and RFA treatment to be performed more safely. One limitation is that the ultrasound contrast agent currently available clinically in Japan is effective for only a short time after injection. It is expected that this limitation will be overcome with the development of new contrast agents.[13]

\section{Conclusions}

The convex puncture probe PVT-350BTP permits percutaneous RFA to be performed safely and effectively.

\section{References}

[1] G. Francica and G. Marone, "Ultrasound-Guided Percutaneous Treatment of Hepatocellular Carcinoma by Radiofrequency Hyperthermia with a "Cooled-Tip Needle": A Preliminary Clinical Experience," European Journal of Ultrasound, Vol. 9, No. 2, 1999, pp. 145-153. doi:10.1016/S0929-8266(99)00022-1

[2] T. Livraghi, N. Goldberg, S. Lazzaroni, et al., "Hepatocellular Carcinoma: Radiofrequency Ablation of Medium and Large Lesions," Radiology, Vol. 214, No. 3, 2000, pp. 761-768.
[3] S. Ross, M. Di Stasi, E. Buscarini, et al., "Percutaneous Ultrasound-Guided Radiofrequency Electrocautery for the Treatment of Small Hepatocellular Carcinoma," Journal of Interventional Radiology, Vol. 8, 1993, pp. 97-103.

[4] N. Hotta, T. Maeno, M. Ayada, et al., "Four-Dimensional Ultrasonography for Therapeutic Radiofrequency Ablation for Hepatocellular Carcinoma," Hepato-Gastroenterology, Vol. 53, No. 70, 2006, pp. 521-525.

[5] N. Hotta, T. Tagaya, T. Maeno, et al., "Advanced Dynamic Flow Imaging with Contrast-Enhanced Ultrasonography for the Evaluation of Tumor Vascularity in Liver Tumors," Clinical Imaging, Vol. 29, No. 1, 2005, pp. 34 41. doi:10.1016/j.clinimag.2004.03.001

[6] T. Teratani, H. Yoshida, S. Shiina, et al., "Radiofrequency Ablation for Hepatocellular Carcinoma in So-Called High-Risk Locations," Hepatology, Vol. 43, No. 5, 2006, pp. 1101-1108. doi:10.1002/hep.21164

[7] K. Katayama, Y. Oosaki, A. Uemura, et al., "Saline Injection into the Pleural Cavity to Detect Tumors of the Hepatic Dome with Sonography: A New Approach for Treatment of Hepatocellular Carcinoma," American Journal of Roentgenology, Vol. 179, No. 1, 2002, pp. 102-104.

[8] N. Hotta, M. Ayda, E. Matsumoto, et al., "Usefulness of Radiofrequency Ablation with Micro-Convex Probe for Hepatocellular Carcinoma," Hepato-Gastroenterology, Vol. 56, No. 93, 2009, pp. 1127-1132.

[9] Y. Matsuda and I. Yabuuchi, "Hepatic Tumors: US Contrast Enhancement with $\mathrm{CO}_{2}$ Microbubbles," Radiology, Vol. 161, No. 3, 1986, pp. 701-705.

[10] S. R. Wilson, P. N. Burns, D. Muradali, et al., "Harmonic hepatic US with Microbubble Contrast Agent: Initial Experience Showing Improved Characterization of Hemangioma, Hepatocellular Carcinoma, and Metastasis," Radiology, Vol. 215, No. 1, 2000, pp. 153-161.

[11] K. Numata, K. Tanaka, T. Kiba, et al., "Contrast- Enhanced, Wide-Band Harmonic Gray Scale Imaging of Hepatocellular Carcinoma: Correlation with Helical Computed Tomographic Findings," Journal of Ultrasound in Medicine, Vol. 20, No. 2, 2001, pp. 89-98.

[12] N. Hotta, T. Tagaya, T. Maeno, et al., "Usefulness of Contrast-Enhanced Ultrasonography with Dynamic Flow Imaging to Evaluate Therapeutic Effects for Hepatocellular Carcinoma," Hepato-Gastroenterology, Vol. 50, No. 54, 2003, pp. 1867-1871.

[13] S. R. Wilson and P. N. Burns, "An Algorithm for the Diagnosis of Focal Liver Masses Using Microbubble Contrast-Enhanced Pulse-Inversion Sonography,' American Journal of Roentgenology, Vol. 186, No. 5, 2006, pp. 1401-1412. doi:10.2214/AJR.04.1920 